\title{
TRAJETÓRIA DOS REQUISITOS UNIFORMES PARA MANUSCRITOS SUBMETIDOS A PERIÓDICOS BIOMÉDICOS*
}

Luciane Favero', Leomar Albini², Elaine Drehmer de Almeida Cruz ${ }^{3}$, Mariluci Alves Maftum4, Maria de Fátima Mantovani ${ }^{3}$, Maria Ribeiro Lacerda ${ }^{5}$

\begin{abstract}
RESUMO: Pesquisa documental com o objetivo de analisar a trajetória dos Requisitos Uniformes para manuscritos submetidos a periódicos biomédicos. Foram analisadas as 13 edições dos Requisitos Uniformes compreendidas entre 1978, ano da primeira edição, a 2010, ano daúltima atualização. Estes Requisitos foram instituídos por um grupo deeditores em Vancouver-Canadáetem como intuito recomendarpadronizações para a apresentação de manuscrito a periódicos científicos daárea da saúde. Foram identificadas várias alterações ocorridas nas versões com a inclusão, revisão ou ampliação de elementos e temas que foram descritos em forma de síntese, com destaque às recomendações vigentes. Os Requisitos auxiliam autores e editores no trabalho de preparação, submissão e divulgação da produção de conhecimento científico naárea da saúde e isso reflete a importância do conhecimento do conteúdo de tal documento, bem como o acompanhamento de suas atualizações. DESCRITORES: Pesquisa; Políticas editoriais; Normas técnicas; Revisão.

\section{TRAJECTORY OF THE UNIFORM REQUIREMENTS FOR MANUSCRIPTS SUBMITTED TO BIOMEDICAL PERIODICALS}

\begin{abstract}
Documentary research whose objective was to analyze the trajectory of the Uniform Requirements for manuscripts submitted to biomedical periodicals. The 13 editions of the Uniform Requirements, covering between 1978, when the first edition was produced, and 2010, the last year of publishing at the time, were analyzed. These requirements were instituted by a group of editors in Vancouver, Canada, and aim to recommend standardizations for the presentation of manuscripts to scientific periodicals in the health area. Various changes were identified which had occurred in the versions, with the inclusion, revision and broadening of elements and themes which were described in the form of a summary, with emphasis on the current recommendations. The Requirements help authors and editors in the work of preparation, submission and dissemination of the production of scientific knowledge in the area of health, and this reflects the importance of knowledge of this document's content-as well as of keeping up with its updates. DESCRIPTORS: Research; Editorial policies; Technical norms; Revision.

\section{TRAYECTORIA DE LOS REQUISITOS UNIFORMES PARA MANUSCRITOS SOMETIDOS A PERIÓDICOS BIOMÉDICOS}

RESUMEN: Investigación documental cuya finalidad fue analizar la trayectoria de los Requisitos Uniformes para manuscritos sometidos a periódicos biomédicos. Fueron analizadas las 13 ediciones de los Requisitos Uniformes comprendidas entre 1978, año de la primera edición, a 2010, año de la última actualización. Estes Requisitos fueron instituidos por un grupo de editores en Vancouver, Canadá y tienen como objetivo recomendar tipificaciones para la presentación de manuscrito a periódicos científicos del área de salud. Fueron identificadas varias alteraciones en las versiones con la inclusión, revisión o ampliación de elementos y temas descriptos en forma de síntesis, con destaque a las recomendaciones vigentes. Los Requisitos ayudan autores y editores en el trabajo de preparación, sumisión y divulgación de la producción de conocimiento científico en el área de la salud y eso muestra la importancia del conocimiento del contenido del documento, así como el acompañamiento de sus actualizaciones. DESCRIPTORES: Investigación; Políticas editoriales; Normas técnicas; Revisión.

*Artigo oriundo da disciplina Análise Crítica de Publicações Científicas da Enfermagem do Curso de Doutorado em Enfermagem do Programa de Pós-Graduação em Enfermagem da Universidade Federal do Paraná - PPGENF UFPR.

${ }^{1}$ Enfermeira.DoutoraemEnfermagem.ProfessoradoCursodeGraduaçãoemEnfermagemdaUniversidadePositivo-UP.MembrodoNúcleodeEstudo, Pesquisa eExtensão em Cuidado Humano deEnfermagem daUFPR-NEPECHE. Membro do Núcleo deEstudos CuidadoeEnsinoem SaúdedaUP. ${ }^{2}$ Enfermeira. Doutoranda pelo Programa de Pós-Graduação em Enfermagem da UFPR - PPGENF. Membro do Grupo de Estudos Multiprofissional em Saúde do Adulto (GEMSA).

${ }^{3}$ Enfermeira. Doutora em Enfermagem. Professora do Departamento de Enfermagem e do PPGENF UFPR. Membro do GEMSA. ${ }^{4}$ Enfermeira.DoutoraemEnfermagem.CoordenadoradoPPGENFUFPR.ProfessoradoDepartamentoedo PPGENFUFPReVice-coordenadoradoNEPECHE. ${ }^{5}$ Enfermeira. Doutora em Enfermagem. Professora do PPGENF UFPR e Professora Visitante do Programa de Pós-Graduação em Enfermagem da Universidade Federal de Santa Maria. Líder do NEPECHE.

Autor correspondente:

Recebido: 23/08/2012

Luciane Favero Aprovado: 03/04/2013

Universidade Positivo

Rua Urbano Lopes, 214 - 80050-520 - Curitiba-PR-Brasil

E-mail: lucianefavero@yahoo.com.br

Cogitare Enferm. 2013 Abr/Jun; 18(2):280-7 


\section{INTRODUÇÃO}

Requisitos Uniformes para Manuscritos (Uniform Requirements for Manuscripts - URM) são padronizações para a elaboração de manuscritos a serem publicados, cuja finalidade é uniformizar a forma de apresentação, além de declararem, entre outras informações, os princípios éticos na conduta e no relato de pesquisa, e de oferecerem recomendações relacionadas a elementos específicos de edição e redação ${ }^{(1)}$. Os URM foram criados com a finalidade de contribuir com autores e editores na tarefa mútua de criar e distribuir relatos precisos, claros e facilmente acessíveis de estudos da área da saúde ${ }^{(2)}$. A utilização das suas recomendações contribui para a qualidade e clareza do relato dos manuscritos submetidos a periódicos da área da saúde e facilita a sua edição ${ }^{(1)}$. Outros grupos, como revisores, editores e leitores em geral, podem ser beneficiados com conhecimentos que elevam a qualidade do processo de pesquisa e redação do texto científico.

O aumento de investigações e publicações na área médica, a preocupação mundial com a condução ética e bioética nos estudos científicos, a criação do Código de Nuremberg e da Declaração de Helsinque, entre outros fatores, incentivaram um pequeno número de editores a se reunirem em Vancouver, Canadá, em 1978 e iniciarem discussão da necessidade de uniformidade para publicações em periódicos científicos na área biomédica. Este grupo ficou conhecido como o "Grupo de Vancouver" o qual, a partir desse encontro, se expandiu até formar o Comitê Internacional de Editores de Revistas Médicas (International Committee of Medical Journal Editors - ICMJE) $)^{(1)}$.

Em 1979, foi publicada a primeira versão dos URM, incluindo formatos de referências bibliográficas, desenvolvidos pela Biblioteca Nacional de Medicina dos Estados Unidos (National Library of Medicine - NLM) ${ }^{(3)}$. Periodicamente o ICMJE se reúne e amplia as discussões de maneira que, atualmente, seu conteúdo extrapola a preparação de originais, resultando em uma série de Pareceres Independentes sobre políticas editoriais. A última versão atualizada dos URM disponível é a de abril de 2010.

Ao compreender a importância dos URM para pesquisadores e editores da área da saúde, incluindo os da Enfermagem, neste artigo o objetivo foi analisar a trajetória dos URM desde o seu início até a última atualização publicada pelo ICMJE.

\section{MÉTODO}

Trata-se de uma pesquisa documental a qual é definida como a observação que objetiva o registro de fenômenos sociais ${ }^{(4)}$. A base documental foi constituída de todas as versões dos URM publicadas, desde a sua criação até a atualidade, as quais estão disponíveis no site do ICMJE, e foram analisadas de setembro a dezembro de $2011 \mathrm{sob}$ a perspectiva da identificação das alterações temporais efetuadas. Vale ressaltar que todo o conteúdo correspondente a cada versão foi analisado integralmente.

\section{RESULTADOS}

Desde a primeira publicação dos URM, em 1979, o ICMJE realizou 13 publicações. Estas foram analisadas, elencadas as principais alterações e contribuições e apresentadas a seguir, por ordem cronológica.

A primeira publicação em 1979 decorreu do $2^{\text {nd }}$ Meeting of the International Steering Committee of Medical Editors, realizado em Montreal-Canadá. O documento apresentou informações para publicações de material científico e contemplou aspectos de preparação e submissão de manuscritos. Entre esses, o espaçamento entre linhas, configuração de margens, número de páginas, página de título, resumo, texto, desenvolvimento, referências, tabelas, legendas, análise estatística e aspectos éticos para pesquisa envolvendo seres humanos ${ }^{(3)}$.

A segunda edição( ${ }^{(5)}$, em 1981, trouxe orientações de publicação prévia ou duplicada, destacando que é responsabilidade dos autores comunicar ao editor, no momento da submissão, todas as publicações prévias do mesmo trabalho ou de outros muito semelhantes, incluindo o envio de cópias que ajudem o editor na avaliação. Apresentou que material rejeitado por uma revista não impedia a submissão a outro periódico. As orientações para citação de referências bibliográficas foram ampliadas e itens referentes às unidades de medida, aos símbolos e abreviações, que constava na versão anterior como apêndices, passaram a fazer parte do corpo do texto.

Em 1988 o ICMJE apresentou a terceira edição dos URM, após reunião realizada em Helsinque-Finlândia, em $1987^{(6)}$. Porém, em texto anterior a esta edição( ${ }^{(7)}$, o Comitê publicou orientações para os erros encontrados nos artigos após divulgação e que exigiam a publicação de correções, ou erratas, de parte do trabalho e das relacionadas a fraudes científicas que requeiram retratação, entre outras medidas. 
A terceira edição ${ }^{(6)}$ trouxe a possibilidade de publicação duplicada, exclusivamente, para edições em idioma diferente do utilizado na primeira publicação do artigo. Nestas situações, as seguinte recomendações deveriam ser observadas: citação, em nota de rodapé, da primeira revista na qual o artigo foi publicado, com referência completa, e observação do intervalo entre as publicações, de pelo menos duas semanas. Estabeleceu critérios de autoria, com ênfase na responsabilidade e contribuição necessárias, além da possibilidade de o editor solicitar a informação da contribuição de cada autor. Em decorrência, o item 'agradecimentos' ganhou mais detalhes e menção, nesse item, das pessoas que não preencham os requisitos de autoria ${ }^{(6)}$. No que se refere às referências, a citação de dissertação e tese passou a ser descrita entre colchetes após o respectivo título ${ }^{(6)}$.

A quarta edição(8), publicada em 1991, recomendou o limite de 150 palavras para resumo não estruturado e 250 para estruturado, e manteve a orientação de uso de três a 10 palavras-chave, provenientes do Medical Subject Headings (MeSH). Esta edição apresentou um quadro que favoreceu o entendimento e ampliou os exemplos e orientações da apresentação das referências; incluiu diretrizes para referenciar materiais audiovisual, não publicado, eletrônico, mapas, dicionários, entre outros.

Em 1994 $4^{(9)}$, nova publicação dos URM, trouxe o acréscimo da seção 'conflito de interesse', recomendou que em artigos coletivos os coautores decidissem a ordem de autoria e destacou que muitas revistas trazem a limitação de número de autores em suas publicações. Sobre a ética incluiu informações de garantia do anonimato aos participantes, principalmente relativo ao uso de fotografias, afirmando que o mascaramento da região ocular em fotografias e alteração dos dados do participante podia ser insuficiente para garantir o anonimato. Porém, se a identificação for inevitável, o consentimento informado deve ser obtido e claramente indicado no texto. Recomendou que as revistas estabelecessem regras editoriais acerca do uso de fotografias nas normas editoriais ${ }^{(9)}$.

Nova publicação ${ }^{(10)}$ foi apresentada à comunidade científica em 1995, sem a seção 'conflito de interesse', que retornou em publicação posterior. Ênfase foi dada à atribuição de autoria em estudos multicêntricos, nos quais todos aqueles nomeados como autores deviam satisfazer plenamente os critérios de autoria. O participante que não atendesse a esses critérios deveria ser listado, porém, com sua permissão, no item agradecimentos ou em apêndice. Houve o acréscimo de recomendação da apresentação de cópia em formato eletrônico (disquete), para artigos em via de serem aceitos nos requisitos de apresentação de manuscritos publicados nas edições anteriores $^{(10)}$.

Em 1997 foi publicada uma edição revisada que novamente enfatizou a aceitação de segunda publicação, principalmente, em outra língua e/ou destinada a um grupo diferente de leitores ${ }^{(11)}$. Houve modificação na orientação da escrita do item 'métodos', com a recomendação da inclusão de detalhes que permitissem a replicação do estudo. Para os estudos de revisão, a forma de localização, seleção, extração e síntese dos dados deviam constar no texto e no resumo. No item referências, foi incluída orientação para manuscritos com mais de seis autores e derivados de estudos multicêntricos ${ }^{(11)}$.

Nova versão (2004) foi disponibilizada no site do $\operatorname{ICMJE}^{(12)}$, em 2004, com a inclusão no corpo do texto dos URM de declarações anteriormente publicadas separadamente. As modificações desta versão, se comparadas às anteriores, se mostram expressivas, pois toda estrutura do documento sofreu reorganização. Houve inclusão de considerações éticas na condução e apresentação do relatório de pesquisa, com detalhamento de critérios de autoria, processo de editoração, revisão por pares, potencial conflito de interesses, privacidade e confidencialidade dos dados, obrigação de divulgação dos resultados negativos, correções e retratações, entre outras, sobre publicações duplicadas e uso de mesma base de dados para pesquisas diferentes ${ }^{(12)}$.

A obrigatoriedade do registro dos ensaios clínicos, citando a Consolidated Standards of Reporting Trials (CONSORT) como forma de apresentação dos relatórios provenientes de ensaios randomizados também marcou a revisão dessa edição ${ }^{(12)}$. Nas orientações gerais sobre o preparo dos manuscritos esta versão inseriu a possibilidade de contagem de palavras do texto e resumo como critério de avaliação; fez evolução no item metodologia e incluiu a possibilidade de submissão online para os periódicos que adotassem essa característica ${ }^{(12)}$.

$\mathrm{Na}$ versão de 2005 o quesito potencial conflito de interesses foi reformulado e, entre outras, recomendou que os autores identificassem a fonte de financiamento, além de continuar relatando potenciais conflitos de interesse ${ }^{(13)}$.

Em fevereiro de 2006, nova atualização ${ }^{(14)}$ sugeriu que as revistas não excluíssem os artigos publicados, mas os mantivessem em seu site ou arquivo, com incentivo de deposição em arquivos múltiplos, para garantir o registro histórico, por meio da preservação dos artigos eletrônicos de forma permanente. Lembrando que 
em caso de correção ou retratação, estas precisariam ser realizadas e publicadas em página citável da edição posterior. Nesta versão foi enfocado, ainda, que os editores poderiam determinar que artigos provenientes de ensaios clínicos tivessem como a obrigatoriedade a colocação, no final do resumo, do número do registro e seu parecer ${ }^{(14)}$.

No item 'preparação e submissão de manuscritos', esta atualização apresentou tabela que orientou autores e editores sobre os relatórios provenientes dos estudos, reportando a alguns órgãos de orientação, dependendo do método utilizado ${ }^{(14)}$. Houve menção de que os autores não deveriam utilizar referências retratadas nos manuscritos, exceto no caso de publicação que se referisse ao assunto ${ }^{(14)}$.

A versão ${ }^{(15)}$ referente ao ano de 2007 não apresentou alterações quando comparada à edição anterior, motivo pelo qual não foi realizada análise de seu conteúdo neste artigo.

Na edição publicada no ano de $2008^{(16)}$ as recomendações para preparação e submissão de manuscritos foram mantidas, mas, na seção intitulada questões editoriais e de publicação relacionadas à publicação em revistas biomédicas foi incluído nos subitens 'correções, retratações e notas de interesse', orientação aos editores quanto às questões relacionadas à improbidade científica e orientação a consultar o fluxograma desenvolvido pela Comissão de Ética em publicação (Committee on Publication Ethics - COPE). Nesta mesma seção, no subitem 'publicação secundária aceitável' a ICMJE esclareceu aos editores de revistas que publicavam simultaneamente artigos em vários idiomas que nos índices da NLM versaria apenas o idioma original, mas todos os demais seriam indicados pelo MEDLINE.

Ainda, quanto às questões prévias ao preparo dos manuscritos, o subitem 'obrigação de registro de ensaios clínicos' versou sobre a importância do registro da metodologia em uma instância pública de registros, porém não apresentou a mesma exigência para registros dos resultados dos ensaios, apesar de haver referência à Food and Drug Administration Amendments Act (FDAAA) ${ }^{(16)}$.

No item relativo ao preparo e submissão dos manuscritos, subitem 'resumo' houve a retirada do termo 'palavras-chave' e de toda a orientação para a utilização dos descritores determinada pelo MeSH. E no item que trata das referências, contemplou a supressão da informação referente ao mês e ao número da publicação do artigo ${ }^{(16)}$.

A versão atual, publicada em 2010, conserva a estrutura da versão anterior, com modificações no texto, sem mudanças no teor central. No subitem 'resumo' além da estrutura de apresentação atualizada na edição de 2008, houve a inclusão da preferência pelos resumos estruturados para pesquisas originais e revisões sistemáticas, e que os resumos de artigos sobre ensaios clínicos incluam os itens considerados essenciais pelo grupo CONSORT, já abordado anteriormente ${ }^{(1)}$.

Vale reforçar que desde a atualização ocorrida em 2008, a padronização de palavras-chave bem como da quantidade dessas não foram mais contemplados nas edições seguintes.

Nesta última edição, no item que aborda as referências, ocorreu a inclusão de instruções para registro de referência disponível por meio eletrônico. Quanto às modificações observadas nesta versão está a ausência de menção do item 'conclusão' ou 'considerações finais' na estrutura do manuscrito, aspecto que foi entendido pelos autores deste estudo como não obrigatório na estrutura textual dos originais submetidos às revistas científicas ${ }^{(1)}$.

Nos Quadros 1 a 3, são apresentadas os principais conteúdos da última edição dos URM quanto às orientações gerais e ao preparo dos manuscritos para submissão.

\section{DISCUSSÃO}

Na análise da evolução dos URM, desde sua primeira edição, constata-se que vários aspectos da publicação inicial estão mantidos, com pouca ou nenhuma alteração no decorrer das atualizações e revisões. Isso pode ser exemplificado pela seção 'resultados e discussão', sobre as quais há orientação que sejam escritas separadamente e sem duplicidade de informações desde a primeira publicação. Da mesma forma, destaca-se a ausência de menção sobre o item 'conclusões ou considerações finais' em todas as versões publicadas. Contempla que na seção discussão devem estar descritas as conclusões do estudo, evitando afirmações não qualificadas e conclusões não apoiadas completamente nos dados. Orienta, ainda, que recomendações, quando for o caso, podem ser incluídas nesse espaço.

As questões éticas estiveram presentes em todas as edições dos URM. Na primeira e segunda publicações consta orientação sobre o seguimento dos preceitos descritos na Declaração de Helsinque, no tópico métodos. A partir da publicação de 1988 esse tema ganhou destaque com a criação de um subitem, específico para as considerações éticas, e a preocupação com o anonimato dos participantes fica explícito. Porém, no ano de 1994, há forte descrição da responsabilidade dos autores sobre a preservação do anonimato dos participantes, necessidade de apresentação de consentimento 
Quadro 1 - Elementos pré-textuais que devem conter em manuscrito submetido a periódico científico conforme recomendação dos Requisitos Uniformes para Manuscritos de 2010. Curitiba, 2012

\begin{tabular}{|l|l|}
\hline \multicolumn{1}{|c|}{ REQUISITO } & \multicolumn{1}{c|}{ DESCRIÇÃO } \\
\hline Princípios gerais & $\begin{array}{l}\text { Conter seções com títulos “IMRAD” (Introdução, Métodos, Resultados e Discussão). } \\
\text { Espaço duplo em todo o texto. } \\
\text { Numeração de páginas, iniciando na página de rosto. }\end{array}$ \\
\hline $\begin{array}{l}\text { Diretrizes para relatório } \\
\text { de estudo }\end{array}$ & $\begin{array}{l}\text { Suprimidas indicações de consultas a Declaração CONSORT. } \\
\text { Incluídas orientações EQUATOR NETWORK (http://www.equator-network.org/ } \\
\text { home/) para relatório de estudo com delineamento específico. }\end{array}$ \\
\hline Página de rosto & $\begin{array}{l}\text { Incluir no título informações que permitirão recuperação eletrônica sensível e es- } \\
\text { pecífica do artigo. } \\
\text { Nome dos autores e afiliações institucionais aos quais o trabalho deve ser atribuído. } \\
\text { Registro de isenção de responsabilidade/propriedade e renúncias se houver. } \\
\text { Autor correspondente (nome, endereço, fone/fax, e-mail). Indicar se o e-mail deve } \\
\text { ou não ser publicado. } \\
\text { Fonte de financiamento. } \\
\text { Título resumido, até 40 caracteres, contando letras e espaços, ao pé da página de rosto. } \\
\text { Contagem de palavras do texto, excluindo resumo, agradecimentos, legendas e } \\
\text { referências. } \\
\text { Menção do número de figuras e tabelas já na página de rosto. }\end{array}$ \\
\hline $\begin{array}{l}\text { Notificação de conflito } \\
\text { de interesses }\end{array}$ & $\begin{array}{l}\text { Constar em página separada, imediatamente após a página de rosto. } \\
\text { Restrumo } \\
\text { tivos, procedimentos básicos (seleção de sujeitos do estudo ou animais de laboratório, } \\
\text { métodos observacionais e analíticos), principais resultados e principais conclusões. } \\
\text { Artigos sobre ensaios clínicos devem conter resumos que incluem itens que o grupo } \\
\text { CONSORT identifica como essenciais (http://www.consort-statement.org/?=1190). }\end{array}$ \\
\hline
\end{tabular}

Fonte: Os autores baseado no Comitê Internacional de Editores de Revistas Médicas ${ }^{(1)}$.

informado e regras para o uso de fotografias, além de aspectos relacionados ao potencial conflito de interesse.

Ressalta-se que, dois anos após a publicação de 1994, foi aprovada no Brasil a Resolução 196/96 do Conselho Nacional de Saúde, que estabelece critérios para as pesquisas envolvendo seres humanos ${ }^{(17)}$. Há que se destacar que o tema relacionado às questões éticas teve ampliada a sua discussão e abordagem no mundo nas últimas duas décadas, principalmente devido as evidências mundiais de abuso de seres humanos em pesquisas ${ }^{(18)}$.

O acompanhamento da evolução mundial, ocorrida na área de produção e divulgação científica, pode ter sido essencial para a inclusão de seções e subitens que não estavam contemplados nas primeiras edições dos URM. Exemplo disso pode ser observado na versão apresentada em outubro de 2004, a qual exibe um layout diferenciado, incorpora pareceres doravante publicados em declarações à parte, inclui orientações referentes às pesquisas clínicas/ensaios clínicos, ao processo de editoração e à possibilidade da submissão online, para os periódicos com formato eletrônico.
A partir da versão apresentada no ano de 2004 foram suprimidos os exemplos de referências bibliográficas, e os URM passaram apenas a apresentar a descrição daquelas utilizadas no documento. Isso é relevante, pois os periódicos utilizados na área da saúde utilizam as orientações do ICMJE e com isso o estilo "Vancouver" das referências. Autores ${ }^{(2)}$ afirmam que dos principais métodos de organização de material bibliográfico, dentro de um texto científico, o estilo Vancouver se tornou o mais difundido e utilizado pelas publicações científicas mundiais.

Com relação às orientações descritas nos Quadros 1 a 3, ressaltavam-se as diretrizes para relatório de estudo com delineamento específico, que orienta os autores a consultarem tais informações nos periódicos escolhidos para a submissão, mas incentiva que se verifiquem as orientações para relatórios na Equator Network ${ }^{(19)}$. Esse centro de recursos se apresenta como uma iniciativa internacional que visava melhorar a confiabilidade e o valor da literatura de pesquisa médica, mediante a promoção de informação transparente e rigorosa dos estudos de investigação ${ }^{(1)}$. 
Quadro 2 - Apresentação dos elementos textuais de manuscrito submetido a periódico científico conforme recomendação dos Requisitos Uniformes para Manuscritos de 2010. Curitiba, 2012

\begin{tabular}{|c|c|}
\hline ELEMENTO & DESCRIÇÃO \\
\hline Introdução & $\begin{array}{l}\text { Fornecer um contexto ou base para o estudo. } \\
\text { O objetivo tem foco mais preciso quando formulado como uma pergunta. } \\
\text { Usar somente referências pertinentes, não incluir dados ou conclusões do trabalho em curso. }\end{array}$ \\
\hline Métodos & $\begin{array}{l}\text { Incluir apenas informações obtidas no momento em que o plano de estudo foi escrito. } \\
\text { Descrever claramente os critérios de seleção dos participantes; apresentar a relevância e jus- } \\
\text { tificar as variáveis como idade, sexo, raça e etnia. } \\
\text { Informações técnicas: método e procedimentos para possível replicação; usar referências de } \\
\text { métodos estabelecidos; fornecer referências de métodos publicados e pouco conhecidos; des- } \\
\text { crever métodos novos ou modificados; justificar seu uso e avaliar suas limitações. } \\
\text { Descrever os métodos estatísticos em detalhes; evitar utilizar somente testes estatísticos de } \\
\text { hipóteses; definir termos estatísticos, abreviaturas e símbolos; especificar o software empregado. }\end{array}$ \\
\hline Resultados & $\begin{array}{l}\text { Apresentar em sequência lógica e em ordem decrescente de importância. } \\
\text { Dados descritos em tabelas ou ilustrações não devem ser repetidos. } \\
\text { Apresentar dados em valores percentuais e absolutos, informar método estatístico de análise. } \\
\text { Usar apenas tabelas e figuras necessárias. } \\
\text { Usar gráficos como alternativa para tabelas com muitos dados. }\end{array}$ \\
\hline Discussão & $\begin{array}{l}\text { Enfatizar aspectos novos e importantes do estudo e as conclusões derivadas dos mesmos. } \\
\text { Dados apresentados anteriormente não devem ser repetidos. } \\
\text { Para estudos experimentais é útil explorar os possíveis mecanismos ou explicações para os } \\
\text { principais achados, comparar e contrastá-los com estudos relevantes, declarar as limitações do } \\
\text { estudo e explorar as implicações dos achados para pesquisas futuras e prática clínica. } \\
\text { Relacionar as conclusões com os objetivos, evitando afirmações absolutas que não tenham } \\
\text { sustentação adequada pelos dados. } \\
\text { Evitar realizar afirmações sobre benefícios econômicos e custos. } \\
\text { Propor novas hipóteses quando justificável. }\end{array}$ \\
\hline Referências & $\begin{array}{l}\text { Considerações sobre as referências seguem formato publicado em 2005, e atualizado em 2006, } \\
2007 \text { e 2008, sem alterações, mas reforça a orientação de que os autores devem consultar } \\
\text { amostras de referências no site do Citing Medicine, mantidos pela NLM. }\end{array}$ \\
\hline
\end{tabular}

Fonte: Os autores baseado no Comitê Internacional de Editores de Revistas Médicas ${ }^{(1)}$.

No espaço virtual indicado no Quadro 1 há, entre outras informações, orientações de como escrever cientificamente um relatório com base no delineamento metodológico, informações sobre o fluxograma de pesquisa. Inclui orientações detalhadas de pontos a serem observados pelos pesquisadores, além de links que orientam relatórios específicos, como é o caso da declaração CONSORT que realiza orientação para ensaios clínicos randomizados ${ }^{(20)}$.

Assim, ao analisar as orientações sugeridas para autores na última edição dos URM, com relação ao preparo dos manuscritos, optou-se por destacar os itens que podem nortear pesquisadores e editores na organização dos originais e também na reestruturação de normas relativas às políticas editoriais das revistas, apresentação dos originais e instrumentos de avaliação dos mesmos.
Convém lembrar que os URM se tratam de recomendações, portanto não têm caráter obrigatório. Assim sendo, cada periódico poderá acatar aquela que julgar conveniente, ou mesmo estabelecer normas próprias que podem diferir das apresentadas no conteúdo dos documentos analisados neste artigo, seguindo formulações específicas e compatíveis com a estrutura, indexação e interesses próprios. Por conseguinte, o autor deverá consultar a política de publicação do periódico no qual intenta publicar seu manuscrito.

Estando os periódicos da Enfermagem inseridos entre aqueles da área biomédica, sobre os quais as recomendações dos URM têm incidência, destaca-se a contribuição que tais mudanças descritas nesse estudo, ocorridas nas versões dos URM, trouxeram para a melhoria da qualidade dos periódicos nacionais de Enfermagem. 
Quadro 3 - Apresentação dos elementos pós textuais de manuscrito submetido a periódico científico conforme recomendação dos Requisitos Uniformes para Manuscritos de 2010. Curitiba, 2012

\begin{tabular}{|c|c|}
\hline Tabelas & $\begin{array}{l}\text { Digitadas ou impressas em folhas separadas em espaço duplo. } \\
\text { Numeradas consecutivamente; nomeadas com um breve título. } \\
\text { Não utilizar linhas horizontais e verticais internas. } \\
\text { Nomear cada coluna com breve título ou abreviado. } \\
\text { Material explicativo deve ser colocado em notas abaixo da tabela, não no título. } \\
\text { Explicar abreviaturas não-padronizadas utilizadas; inclui nas legendas *,†, }+\|,\|, \oplus, *,+\dagger,+ \text {, }+ \text {, etc. } \\
\text { Identificar medidas estatísticas de variação (desvio padrão, erro padrão da média). } \\
\text { Certificar-se que cada tabela é mencionada no texto. }\end{array}$ \\
\hline Ilustrações & $\begin{array}{l}\text { Desenhadas ou fotografadas profissionalmente ou submetidas como impressões digitais de } \\
\text { qualidade fotográfica. } \\
\text { Enviar arquivo eletrônico das figuras em formato (JPEG, GIF, por exemplo). } \\
\text { Raio X, ultrassonografias ou outras imagens diagnósticas, enviar fotografias nítidas em papel } \\
\text { brilhante, branco e preto ou coloridas. Tamanho da fotografia: } 5 \text { X } 7 \text { polegadas. } \\
\text { Autoexplicativas, com títulos e explicações nas legendas e não nas ilustrações. } \\
\text { Fotos de pessoas não devem ser identificáveis, ou quando nesta condição necessitam ter per- } \\
\text { missão escrita para esse uso. } \\
\text { Numeradas consecutivamente conforme ordem de aparecimento no texto. } \\
\text { Para figuras previamente publicadas, deve-se dar crédito à fonte original ou pedir permissão } \\
\text { para reprodução (exceto domínio público). } \\
\text { Para ilustrações coloridas, verificar exigências da revista. } \\
\text { Figuras em formato eletrônico devem seguir normas da revista. }\end{array}$ \\
\hline $\begin{array}{l}\text { L e g e n d a s } \\
\text { para } \\
\text { Ilustrações } \\
\end{array}$ & $\begin{array}{l}\text { Em espaço duplo, página separada, com algarismos arábicos correspondentes. } \\
\text { Identificar e explicar na legenda símbolos, setas ou letras utilizados. } \\
\text { Explicar a escala interna e o método de coloração nas foto micrografias. }\end{array}$ \\
\hline $\begin{array}{l}\text { Unidades de } \\
\text { Medida }\end{array}$ & $\begin{array}{l}\text { Medições de largura, altura, peso e volume devem ser relatados em unidades métricas (metro, } \\
\text { kilograma, litro, etc) ou em múltiplos decimais. } \\
\text { Temperatura (em graus Celsius); pressão sanguínea (em milímetros de mercúrio), ou outras } \\
\text { especificadas pela revista. }\end{array}$ \\
\hline $\begin{array}{l}\text { Abreviaturas } \\
\text { e símbolos }\end{array}$ & $\begin{array}{l}\text { Utilizar somente abreviaturas padronizadas; evitar abreviaturas no título. } \\
\text { Termos abreviados devem ser escritos por extenso, seguidos da abreviatura na primeira vez } \\
\text { que aparecem no texto. }\end{array}$ \\
\hline
\end{tabular}

Fonte: Os autores baseado no Comitê Internacional de Editores de Revistas Médicas ${ }^{(1)}$.

\section{CONCLUSÃO}

Esta pesquisa teve como objetivo analisar as edições dos URM e permitiu observar que as recomendações de padronização, para a submissão de manuscritos decorrente de mais de 30 anos de elaborações e atualizações realizadas pelo ICMJE, auxiliam editores, autores/pesquisadores da área da saúde, comunidade científica em âmbito internacional no trabalho e compromisso da divulgação e socialização da produção de conhecimento científico.

Mundialmente os URM têm ampla utilização pelos periódicos científicos em suas políticas editoriais e suas recomendações de padronizações constituem-se ferramenta indispensável para a orientação de autores e editores. Portanto, é imperioso que pesquisadores, editores, estudantes e demais interessados em pesquisa e publicação científica conheçam os princípios descritos pelo referido documento, bem como fiquem atentos às atualizações periódicas realizadas pelo ICMJE, que contribuirão na empreitada de organizar e submeter um manuscrito a periódico científico.

\section{REFERÊNCIAS}

1. International Committee of Medical Journal Editors (ICMJE): uniform requirements for manuscripts submitted to biomedical journals: writing and editing for biomedical publication. [Internet] 2010 [acesso em 30 out 2011]. Disponível: http://www.icmje.org/urm_full.pdf 
2. Rother ET, Braga MER. O novo estilo de Vancouver: o que mudou nas referências. Arq. bras. oftalmol. [Internet] 2004;67(4) [acesso em 15 mai 2012]. Disponível: http:// dx.doi.org/10.1590/S0004-27492004000400025.

3. International Steering Committee of Medical Editors. Uniform requirements for manuscripts submitted to biomedical journals. Br. Med. J. BMJ. [Internet] 1979;1 [acesso em 30 out 2011]. Disponível: http://www.icmje. org/1979_urm.pdf

4. Richardson RJ, Peres JAS, cols. Pesquisa social: métodos e técnicas. São Paulo: Atlas; 1999.

5. International Committee of Medical Journal Editors (ICMJE). Uniform requirements for manuscripts submitted to biomedical journal. Br. Med. J. BMJ. [Internet] 1982;284(12) [acesso em 30 out 2011]. Disponível: http://www.icmje.org/1982_urm.pdf

6. International Committee of Medical Journal Editors (ICMJE). Uniform requirements for manuscripts submitted to biomedical journal. Br. Med. J. BMJ. [Internet] 1988;(296) [acesso em 30 out 2011]. Disponível: http://www.icmje.org/1988_urm.pdf

7. International Committee of Medical Journal Editors (ICMJE). Retraction of research findings. $\mathrm{Br}$. Med. J. BMJ. [Internet] 1988;(296) [acesso em 30 out 2011]. Disponível: http://www.icmje.org/urm_ statement_1988.pdf

8. International Committee of Medical Journal Editors (ICMJE). Uniform requirements for manuscripts submitted to biomedical journal. Br. Med. J. BMJ. [Internet] 1991;302(9) [acesso em 30 out 2011]. Disponível: http://www.icmje.org/1991_urm.pdf

9. International Committee of Medical Journal Editors (ICMJE). Uniform requirements for manuscripts submitted to biomedical journal. Can Med Assoc J [Internet] 1994;150(2) [acesso em 30 out 2011]. Disponível: http://www.icmje.org/1994_urm.pdf

10. International Committee of Medical Journal Editors (ICMJE). Uniform requirements for manuscripts submitted to biomedical journal. Can Med Assoc J [Internet] 1995;152(9) [acesso em 30 out 2011]. Disponível: http://www.icmje.org/1995_urm.pdf

11. International Committee of Medical Journal Editors (ICMJE). Uniform requirements for manuscripts submitted to biomedical journal. N Engl J Med [Internet] 1997;336(4) [acesso em 30 out 2011]. Disponível: http:// www.icmje.org/1997_urm.pdf
12. International Committee of Medical Journal Editors (ICMJE). Uniform requirements for manuscripts submitted to biomedical journals: writing and editing for biomedical publication. [Internet] 2004 [acesso em 30 out 2011]. Disponível: http://www.icmje.org/2004_ urm.pdf

13. International Committee of Medical Journal Editors (ICMJE). Uniform requirements for manuscripts submitted to biomedical journal. [Internet] 2005 [acesso em 30 out 2011]. Disponível: http://www.icmje. org/2005_urm.pdf

14. International Committee of Medical Journal Editors (ICMJE). Uniform requirements for manuscripts submitted to biomedical journals: writing and editing for biomedical publication. [Internet] 2006 [acesso em 30 out 2011]. Disponível: http://www.icmje.org/2006 urm.pdf

15. International Committee of Medical Journal Editors (ICMJE). Uniform requirements for manuscripts submitted to biomedical journals: writing and editing for biomedical publication. [Internet] 2007 [acesso em 30 out. 2011]. Disponível: http://www.icmje.org/2007_ urm.pdf

16. International Committee of Medical Journal Editors (ICMJE). Uniform requirements for manuscripts submitted to biomedical journals: writing and editing for biomedical publication. [Internet] 2008 [acesso em 30 out 2011]. Disponível: http://www.icmje.org/2008_ urm.pdf

17. Junior KF. Pesquisa em saúde: ética, bioética e legislação. Goiânia: AB; 2003.

18. Fernandes MR, Queiroz MCCAM, Moraes MR, Barbosa MA, Souza ALL. Padrões éticos adotados pelas revistas científicas brasileiras das especialidades médicas. Rev. Assoc. Med. Bras. [Internet] 2011;57(3) [acesso em 15 mai 2012]. Disponível: http://www.scielo. br/pdf/ramb/v57n3/v57n3a07.pdf

19. Equator Network. Enhancing the quality and transparency of health research. [Internet] [acesso em 02 dez 2011]. Disponível: http://www.equator-network. org

20. Consolidated Standards of Reporting Trials (CONSORT). Transparent reporting of trials. [Internet] [acesso em 02 dez 2011]. Disponível: http://www. consort-statement.org/? $=1190$ 